\title{
Influence de la température sur l'accumulation par la voie directe du 60Co chez un poisson dulçaquicole Influence de la température sur l'accumulation par la voie directe du ${ }^{60}$ Co chez un poisson dulçaquicole
}

\author{
J. P. Baudin et A. F. Fritsch
}

Volume 1, numéro 4, 1988

URI : https://id.erudit.org/iderudit/705018ar

DOI : https://doi.org/10.7202/705018ar

\section{Aller au sommaire du numéro}

\section{Éditeur(s)}

Université du Québec - INRS-Eau, Terre et Environnement (INRS-ETE)

\section{ISSN}

0992-7158 (imprimé)

1718-8598 (numérique)

Découvrir la revue

Citer cet article

Baudin, J. P. \& Fritsch, A. F. (1988). Influence de la température sur l'accumulation par la voie directe du 60Co chez un poisson dulçaquicole. Revue des sciences de l'eau / Journal of Water Science, 1(4), 385-400. https://doi.org/10.7202/705018ar

\section{Résumé de l'article}

L'influence du facteur thermique sur la contamination de la carpe par le ${ }^{60} \mathrm{Co}$ a été étudiée pour 4 températures : $8^{\circ} \mathrm{C}, 15^{\circ} \mathrm{C}, 20^{\circ} \mathrm{C}$ et $25^{\circ} \mathrm{C}$. Pour chaque expérience, un lot homogène de 10 individus, d'une masse initiale d'environ 2,3 $\mathrm{g}$ et nourris ad libitum, est introduit dans 5 litres d'eau, renouvelée tous les 3 à 4 jours et contaminée à raison de $1,2.10^{5} \mathrm{~Bq} . \mathrm{l}-1$.

Entre deux renouvellements, la concentration $\mathrm{du}^{60} \mathrm{Co}$ dans l'eau décroît sensiblement et l'état ionique du radionucléide, introduit à $100 \%$ sous forme cationique, évolue vers des formes anioniques et neutres. Ces deux faits sont liés au métabolisme des poissons dont la croissance pondérale est corrélée à la température, la distribution de la nourriture étant adaptée aux besoins des individus.

Globalement, la quantité totale de radiocobalt fixée par les carpes apparaît très fortement liée au facteur thermique mais, selon le niveau des températures, des situations différentes se dégagent pour des mêmes écarts. Il n'y a pas proportionnalité comptéte entre élévation de température et accumulation du ${ }^{60}$ Co mais une augmentation discontinue témoignant de l'existence de paliers, dont le plus important se situe à $20^{\circ} \mathrm{C}$.Le facteur de concentration du ${ }^{60} \mathrm{Co}$ est, par contre, peu influencé par le facteur thermique pour les écarts inférieurs à $10^{\circ} \mathrm{C}$ ou quand la température maximale est inférieure à $20^{\circ} \mathrm{C}$. L'incidence de la température devient sensible quand les écarts dépassent $10^{\circ} \mathrm{C}$ et notamment lorsque sont atteintes des valeurs correspondant à l'optimum thermique des carpes. A l'équilibre, le facteur de concentration moyen du ${ }^{60} \mathrm{Co}$ est de 3,6 à 8 ${ }^{\circ} \mathrm{C}, 4,4$ à $15^{\circ} \mathrm{C}, 5,1$ à $20^{\circ} \mathrm{C}$ et 6 à $25^{\circ} \mathrm{C}$. 


\title{
Influence de la température sur l'accumulation par la voie directe du ${ }^{60} \mathrm{Co}$ chez un poisson dulçaquicole
}

\author{
Temperature influence upon the accumulation of \\ ${ }^{60} \mathrm{Co}$ by direct way in \\ a freshwater fish
}

RÉSUMÉ

L'influence du facteur thermique sur la contamination de la carpe par le ${ }^{60} \mathrm{Co}$ a été étudiée nour 4 températures : $8{ }^{\circ} \mathrm{C}$, $15{ }^{\circ} \mathrm{C}, 20{ }^{\circ} \mathrm{C}$ et $25^{\circ} \mathrm{C}$. Pour chaque expérience, un lot homogène de 10 individus, d'une masse initiale d'environ $2,3 \mathrm{~g}$ et nourris ad libitum, est introduit dans 5 litres d'eau, renouvelée tous les 3 à 4 jours et contaminée à raison de $1,2 \cdot 10^{5} \mathrm{~Bq} \cdot \mathrm{L}^{-1}$.

Entre deux renouvellements, la concentration du ${ }^{60}$ co dans l'eau décroît sensiblement et l'état ionique du radionucléide, intrcduit a $100 \%$ sous forme cationique, èvolue vers des formes anioniques et neutres. Ces deux faits sont liés au métabolisme des poissons dont la croissance pondérale est corrélée à la température, la distribution de la nourriture étant adaptée aux besoins des individus.

Globalement, la quantité totale de radiocobalt fixée par les carpes apparait très fortement tiée au facteur thermique mais, selon le niveau des temperatures, des situations différentes se dénagent pour des mêmes écarts. Il n'y a pas proportionnalité complète entre élévation de température et accumulation du ${ }^{60}$ co mais une augmentation discontinue témoignant de l'existence de paliers, dont le plus important se situe à $20^{\circ} \mathrm{C}$.

(*) Chargé de Recherche au C.N.R.S.

(1) Laboratoire de Radioécologie des Eaux Continentales, DERS/SERE CEN CADARACHE, 13108 Saint-Pau1-lez-Durance Cédex, France.

Té1. : 42-25-26-15. 
Le facteur de concentration du ${ }^{60}$ Co est, par contre, peu influencé par le facteur thermique pour les écarts inférieurs à $10{ }^{\circ} \mathrm{C}$ ou quand la température maximale est inférieure à $20^{\circ} \mathrm{C}$. L'incidence de la température devient sensible quand Les écarts dépassent $10^{\circ} \mathrm{C}$ et notamment lorsque sont at teintes des valeurs correspondant à l'optimum thermique des carpes. A l'équilibre, le facteur de concentration moyen du ${ }^{60} \mathrm{Co}$ est de 3,6 à $3{ }^{\circ} \mathrm{C}, 4,4$ à $15^{\circ} \mathrm{C}, 5,1$ à $20^{\circ} \mathrm{C}$ et 6 à $25^{\circ} \mathrm{C}$.

Mots-clés: ${ }^{60} \mathrm{Co}$, poisson, eau douce, bioaccurulation, température, facteur de concentration.

The problems of simultaneous thermal and radionuclide pollutions have led to a number of laboratory and field studies of the temperature effects on the radioactive contamination of aquatic organisms. AMIARD and KHALANSKI (1981) concluded from their bibliographic study that a temperature rise generally has a positive effect on biological accumulation of radionuclides in freshwater organisms. Due to insufficient data, however, this conclusion does not appear to be substantiated for ${ }^{60} \mathrm{co}$ uptake by fish. An experimental study was therefore undertaken to assess the effect of temperature on ${ }^{60}$ Co uptake by Cyprinus carpio.

The importance of ${ }^{60} \mathrm{Co}$ results from the fact that, apart from tritium, radioactive cobalt isotopes represent the major components of liquid wastes discharged from pressurized water reactors, which are widely operated in France (BARDIN et al., 1982 ; PICAT et al., 1986). The common carp (Cyprinus carpio L.) was selected as the subject of this experimentation because of its wide geographic distribution and the frequency with which it is found in field samples, making it a suitable biological indicator (D'AUBENTON and SPILLMANN, 1978 ; FOULQUIER and PALLY, 1982 ; FOULQUIER et al., 1935).

The effect of temperature on ${ }^{60} \mathrm{Co}$ contamination of the carp was investigated by conducting the same experiment at four temperatures $\left(8^{\circ} \mathrm{C}, 15^{\circ} \mathrm{C}, 20^{\circ} \mathrm{C}\right.$ and $25^{\circ} \mathrm{C}$ ) determined by ecological and physiological criteria. In each experiment, young carps with a mean weight of $2.3 \mathrm{~g}$ were placed in a tank containing 5 liters of water contaminated with $1.2 \times 10^{5} \mathrm{~Bq} . \mathrm{L}^{-1}$ of ${ }^{60} \mathrm{Co}$. The water was renewed at intervals of $3-4$ days. Modifications in the ionic form of the radioactive cobalt dissolved in the water were monitored by percolating filtered water samples through ion exchange resin columns. The carps were fed to satiation with an industrial food containing $12 \%$ minerals, $8 \%$ fats and $53 \%$ proteins (dry weight) together with vitamins and trace elements including $0.2 \mathrm{mg}-\mathrm{kg}^{-1}$ of cobalt. Radioactivity levels in the fish were measured, after mild anesthesia, with a single-channel amplitude selector equipped with a thallium-activated flat sodium iodine orcbe.

Between successive water renewals, the ${ }^{60}$ Co concentration diminished (Table 1 ) and the ionic form of the radionuclide remaining in solution was significantly modified. The ${ }^{60} \mathrm{Co}$ was 
introduced in chloride form and was initially found exclusively as a cation; anionic or neutral forms appeared and became dominant after a few days (Figure 1 ). These two phenomena are probably related to the fish metabolism which is temperaturedependent. Thus, food was distributed according to the individual requirements, and an exponential correlation was observed between the temperature and the weight gain in the carps (Table 1). The appearance of anionic and neutral forms may be attributed to excessive amounts of organic matter due to the presence of the fish and food (FUKAI and MURRAY, 1973 ; KILLEY et al., 1984). This evolution also results from a significant increase in ions such as $\mathrm{HCO}_{3}^{-}, \mathrm{Cl}^{-}, \mathrm{SO}_{4}{ }^{--}, \mathrm{PO}_{4}{ }^{--}$and $\mathrm{NH}_{4}{ }^{+}$(FRITSCH and BAUDIN, 1984) which may combine with $\mathrm{CO}^{++}$ions to form neutral molecules and anionic complexes (TRISCHAN et al., 1981). Radioactive decay in the water is also temperature-dependent : the amount of fecal matter, which strongly adsorbs radioactive cobalt, is proportional to the metabolic intensity in the fish.

The overall ${ }^{60} \mathrm{Co}$ uptake by the carps (Table 2) appears to be strongly related to the temperature, but the effect of a given temperature rise varied with the initial temperature (Table 4). The ${ }^{60}$ Co uptake was not fully proportional to the temperature rise, but was observed to increase in stepwise tashion indicating the existence of various thresholds. The most significant of these was noted at $20^{\circ} \mathrm{C}$, which is the lower limit of the optimum temperature range for carps.

The ${ }^{60} \mathrm{Co}$ concentration in th fish (Tabte 3 ) was influenced to a much lower extent by the temperature than the total uptake. Small temperature variations $\left(5-7^{\circ} \mathrm{C}\right)$ had little or no effect on the concentration factor; this was also true for larger variations, from $8{ }^{\circ} \mathrm{C}$ to $20{ }^{\circ} \mathrm{C}$ (Table 4). Temperature effects were only significant between $8{ }^{\circ} \mathrm{C}$ and $25^{\circ} \mathrm{C}$, and between $15{ }^{\circ} \mathrm{C}$ and $25^{\circ} \mathrm{C}$.

The ${ }^{60}$ Co concentration variation in time was similar for all four temperatures, with two successive phases (Figure 2). It can be expressed mathematically by an exponential model based on the existence of several compartments in living organisms characterized by the influx. and efflux rates for the element under consideration (GOLSTEIN and ELWOOO, 1971 ; AOYAFA and INOUE, 1973 ; PENTREATH, 1975 ; GONTIER, 1983). The maximum value and the time required to reach equilibrium were determined by extrapolation from the equations for the concentration factor variations. The results were 3.6 and 310 days at $8{ }^{\circ} \mathrm{C}$, 4.4 and 220 days at $15{ }^{\circ} \mathrm{C}, 5.1$ and 125 days at $20{ }^{\circ} \mathrm{C}$, and 6 and 110 days at $25^{\circ} \mathrm{C}$. These vatues indicate that a temperature rise diminishes the time required to reach equilibrium and slightly increases the concentration factor by an amount that is statistically significant only for major temperature differences (Table 4).

Detailed investigations of various freshwater species, notably Cyprinus carpio, have demonstrated the decisive effect of temperature on metabolic activity in these animals (LEVESQUE, 1986). The results presented here indicate that the ${ }^{60}$ Co uptake rate in the carp is not proportional to the temperature rise as is generally the case for physiological processes. From a radiation prctection standpoint, this observation suggests that the 
temperature is not an essential factor in ${ }^{60} \mathrm{Co}$ contamination of freshwater fish.

Key-words : "Co, fish, freshwater, bioaccumulation, temperature, concentration factor.

\section{INTRODUCTION}

En raison d'une possible simultanéité des pollutions thermique et radioactive, l'influence de la température sur la contamination des organismes aquatiques, par des radionucléides, a donné lieu à divers travaux expérimentaux ainsi qu'a des constats in situ. A l'issue d'une etude bibliographique, AMIARD et KHALANSKI (1981), concluent à un effet généralement positif de l'élévation de la température sux la bioaccumulation des radionucléides par les organismes dulçaquicoles. Pour les poissons toutefois, cette conclusion ne nous parait pas totalement satisfaisante, dans la mesure où les données disponibles sont relativemert peu nombreuses et tirées d'expériences ou d'observations effectuées dans des conditions très disparates.

En ce qui concerne plus particulièrement les radiocobalts, on constate que le nombre de travaux relatifs aux poissons dulçaquicoles est réduit, et que les études traitant de l'influence de la tempéxature sont très rares (PHILLIPS et RUSSO, 1978 ; AMIARD et KHALANSKI, 1981 ; COLE et CARSON, 1981 ; COUGHTREY et THORNE, 1983). Or, comme cela a été mis en évidence par plusieurs auteurs, les cobalts 58 et 60 sont les constituants majeurs, hors tritium, des effluents liquides rejetés par les réacteurs à eau pressurisée (KAYE, 1973 ; BARDIN et al., 1982 ; PICAT et al., 1986). En France, cette filière est largement répandue avec 43 réacteurs en fonctionnement le ler janvier 1987 et une douzaine d'autres devant être mis en service dans les 5 années suivantes. En raison des sites d'implantation des centrales électronucléaires, les eaux douces sont particuliẻrement concernées par les radiocobalts, corme le démontrent les mesures effectuées sur des échar:tillons de sédiments et de faune prélevés dans la Meuse et le Rhóne, par exemple (CANTILLoN et al., 1969 ; MICHOLET-COTE et al., 1973 ; FOULQUIER et PALLY, 1984 ; FOULQUIER et al., 1985). Cette importance, nous a conduit à élaborer un programne de travaux expérimentaux avec le ${ }^{60} \mathrm{Co}$, qui comporte l'étude de l'influence de la température sur la radiocontamination de la carpe dont les résultats font l'objet de la présente note.

La carpe commune; Cyprinus carpio L., a été choisie en raison de sa très large répartition gégraphique et de sa fréquence dans les prélèvements in situ, qui confèrent à cette espèce le caractère d'indicateur radiohydrobiologique (MUUS et DALHSTROM, 1968 ; D'AUBENTON et SPILLMANN, 1976 ; FOULQUIER et PALIY, 1982 ; FOULQUIER et al., 1985). 
L' influence du facteur thermique sur 1 'accumulation du ${ }^{6}$ Co par la carpe a été étudiée en réalisant la même expérience, dans des conditions identiques, mais à 4 températures, $8{ }^{\circ} \mathrm{C}, 15^{\circ} \mathrm{C}, 20^{\circ} \mathrm{C}$ et $25^{\circ} \mathrm{C}$, déterminêes selon des critères physiologiques et écologiques. La température minimale $\left(8^{\circ} \mathrm{C}\right)$ représente un seuil de subsistance en milieu naturel (MUUS et DALHSTROM, 1968). Au cours d'un test préliminaire, nous avons vérifié qu'elle correspondait bien à la température au-dessous de laquelle les carpes cessaient de $s^{\prime}$ alimenter. Les trois autres constituent les différentes limites des optima thermiques des carpes, qui concernent la nutrition, la croissance et la reproduction (MUUS et DALHSTRCM, 1968 ; HUET, 1970 ; D'AUBENTON et SPILLMANN, 1976). Du point de vue écologique, les quatre températures choisies entrent dans la gamme de celles que l'on peut relever, en fonction des saisons, dans de grands fleuves français comme la seine et le Rhône (LACOMBE et CREACH, 1974 ; MURAT et PARENT, 1974 ; DESSAIX, 1980).

Pour chacune des températures, l'expérience est réalisée sur un lot de 10 carpes juvéntles d'environ $2,3 \mathrm{~g}$, placées dans un aquarium contenant 5 litres d'eau de source contaminée à raison d'environ $1,2 \cdot 10^{5} \mathrm{~Bq} \cdot 1^{-1}$ de ${ }^{60} \mathrm{Co}$. La concentration du cobalt stable qui est infërieure à $0,2 \mu g \cdot 1^{-1}$ n'est pas modifiée lors de la contamination de $l^{\prime}$ eau, la solution de ${ }^{6} \mathrm{CoCl}_{2}$ utilisée étant dépourvue d'entraineur. L'eau est renouvelée 2 fois par semaine (Mardi et Jeudi), le niveau initial de la radioactivité étant toujours respecté. Ia répétition de cette opération tout au long de l'expérience, simule une contamination aigũe du milieu mais restitue également une contamination chronique, la concentration en ${ }^{60} \mathrm{Co}$ de $l^{\prime}$ eau restant toujours superieure à $0,5 \cdot 10^{5} \mathrm{~Bq} \cdot 1^{-1}$. Les modifications de la forme ionique du radiocobalt dans les bacs d'expérimentation sont déterminées par la percolation, lors de chaque renolvellement, d'un échantilion de $20 \mathrm{ml}$ d'eau, préalablement filtrếe. Le dispositif de percolation est composé de 3 colonnes placées en série, la première constituée de résine êchangeuse d'ions positifs, la seconde de résine échangeuse d'ions négatifs et la troisième d'adsorbants synthétiques neutres.

Les carpes sont nourries ad libitum, 5 jours par semaine, avec un aliment industriel, distribué sous forme de granulés, renfermant 12 \% de matiéres minérales, 8 \& de lipides et 58 \& de protéines (par rapport a la matière sèche), ainsi que des vitamines et des oligoéléments, dont $0,2 \mathrm{mg} . \mathrm{kg}^{-1}$ de cobalt.

La radioactivité des échantillons est mesurée avec un sélecteur d'amplitude monocanal dont le dispositif de détection est une sonde d'iodure de sodium active au thallium. Pour les poissons, les mesures sont effectuées après une légère anesthésie, pratiquée par un bain dans une solution de MS 222 SANDOZ à $0,3 \mathrm{~g} \cdot \mathrm{I}^{-1}$, operation qui permet une parfaite reproductibilité de la géométrie pour la détermination de la radioactivité. Le facteur de concentration du ${ }^{60}$ co est calculé avec des concentrations moyennes du radionucléide dans l'ear évaluêes d'après les valeurs initiales et celles mesurées au moment de chaque renouvellement (de 101 à $107 \mathrm{~Bq} \cdot \mathrm{ml}^{-1}$ selon la température). Dans les tableaux, les résultats sont gênéralement donnés sous la forme de valeurs moyennes affectées de leur intervalle de confiance calculé selon la formule statistique t.s $/ \sqrt{n}$ dans laquelle " $t$ " est la variable de student pour 95 de probabilité, "s" la déviation standard estimée et " $n$ " le nombre de mesures. Dans le cas des données concernant les carpes, ce nombre "n" est égal à 10 . 


\section{RÉSULTATS ET DISCUSSIONS}

Paramētres relatifs au milieu

Quelle que soit la température, le pH de l'eau évolue assez peu au cours des cycles que constituent les périodes séparant deux renouvellements successifs de l'eau des aquariums (tableau 1). Dans le même temps, la concentration du ${ }^{60}$ Co décrolt assez sensiblement, en raison de sa fixation par les poissons mais essentiellement à cause de son adsorptior. sur les fèces émises par les poissons, sur le diffuseux d'air et les paxois des bacs. On constate que cette dëcroissance est partiellement proportionnelle à la temperature, la perte de radioactivité étant de 19,7 \& à $8{ }^{\circ} \mathrm{C}, 22,9$ \& à $15^{\circ} \mathrm{C}, 27,3$ \& à $20^{\circ} \mathrm{C}$ et 25,9 a à $25^{\circ} \mathrm{C}$.

Tableau 1.- Principaux paramètres expérimentaux.

Table 1.- Main experimental parameters.

\begin{tabular}{|c|c|c|c|c|}
\hline \multirow{2}{*}{ Paramètres } & \multicolumn{4}{|c|}{ Températures } \\
\hline & $8^{\circ} \mathrm{C}$ & $15^{\circ} \mathrm{C}$ & $20^{\circ} \mathrm{C}$ & $25^{\circ} \mathrm{C}$ \\
\hline Durée de l'expérience (jours) & 54 & 54 & 43 & 49 \\
\hline pH moyen de l'eau ${ }^{(1)}$ & $7,7 \pm 0.1$ & $7,7 \pm 0,1$ & $7,5 \pm 0.1$ & $7,6 \div 0,1$ \\
\hline Concentration initiale $^{(2)}$ & $117 \pm 3$ & $118 \pm 3$ & $117 \pm 3$ & $116 \pm 3$ \\
\hline $\begin{array}{l}\text { du }{ }^{60} \text { Co dans } \\
\text { l'eau (Bq.ml) }\end{array}\left\{\begin{array}{l}\text { au renouvellement } \\
\text { moyenne }\end{array}\right.$ & $\begin{array}{r}94 \pm 13 \\
107 \pm 8\end{array}$ & $\begin{aligned} 91 & \pm 11 \\
105 & \pm 7\end{aligned}$ & $\begin{array}{r}85 \pm 10 \\
101 \pm 8\end{array}$ & $\begin{array}{r}86 \pm 10 \\
101 \pm 7\end{array}$ \\
\hline Masse moyenne f initiale & $2,5 \pm 0,3$ & $2,2 \pm 0,2$ & $2,2 \pm 0,2$ & $2,4 \pm 0,3$ \\
\hline des carpes $(g)\{$ finale & $2,6 \pm 0,3$ & $3,1 \pm 0,3$ & $4,5 \pm 0,4$ & $5.9 \pm 0,7$ \\
\hline $\begin{array}{l}\text { Equation de l'évolution de la masse } \\
\text { des carpes }\end{array}$ & $\begin{array}{l}2,4 \cdot e^{0,001 t} \\
\left(r^{2}=0,81\right)\end{array}$ & $\begin{array}{l}2,1 \cdot \mathrm{e}^{0,007 t} \\
\left(\mathrm{r}^{2}=0,96\right\}\end{array}$ & $\begin{array}{l}2,2 \cdot e^{0,018 t} \\
\left(r^{2}=0,98\right)\end{array}$ & $\begin{array}{c}2,4 . e^{0,020 t} \\
\left(r^{2}=0,99\right)\end{array}$ \\
\hline $\begin{array}{l}\text { Taux de grossissement journalier des } \\
\text { carpes }\left\{m g . j^{*} \text { \} }\right.\end{array}$ & 2,4 & 17,4 & 52,8 & 73 \\
\hline
\end{tabular}

Moyennes et déviations standard calculées pour $(1)_{37}$ et $(2)_{15}$ valeurs.

En raison du protocole adopté, l'évolution ionique du ${ }^{6}{ }^{\circ} \mathrm{Co}$ est suivie sur des périodes de 3 ou 4 jours et cette étude est répétée 13 ou 14 fois selon l'expérience. Le radiocobalt est introduit à l'état de chlorure et se trouve initialement en totalité sous forme cationique. Le bilan ionique du ${ }^{60} \mathrm{Co}$ restant dissolis dans $1^{\prime}$ eau, établi à la fin de chaque cycle, révèle une évolution très nette : des formes anioniques et neutres appataissent et les formes cationiques deviennent minoritaires. Toutefois, on peut distinguex deux situations en fonction de la température. Dans le bac maintenu à $8^{\circ} \mathrm{C}$, la transformation est limitée, les formes cationiques sont les plus importantes et représentent en moyenne 45 (figure $1 \mathrm{~A}$ ). 
Dans les 3 autres cas, au contraire, les formes anioniques sont largement majoritaires et représentent de 55 à 60 \& du radionucléide dissous dans l'eau (figures 1B, C et D).

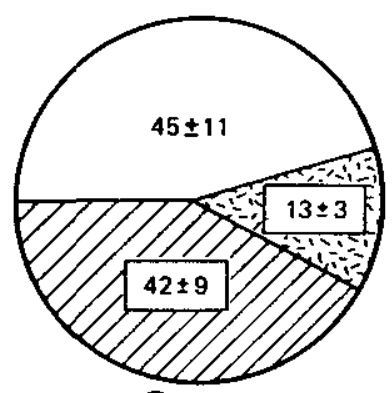

(A) $8{ }^{\circ} \mathrm{C}$

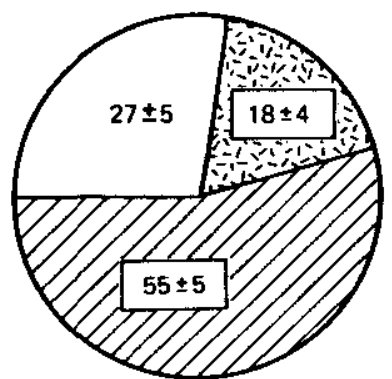

(c) $20^{\circ} \mathrm{C}$

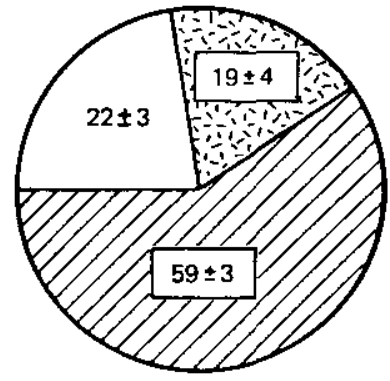

(B) $15^{\circ} \mathrm{C}$

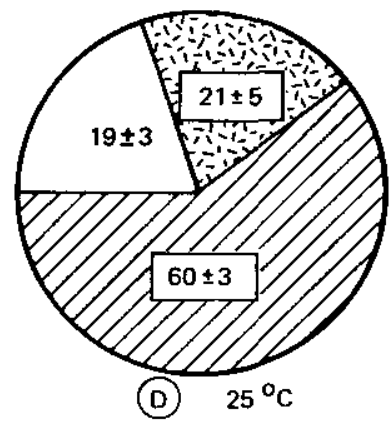

(D) $25^{\circ} \mathrm{C}$

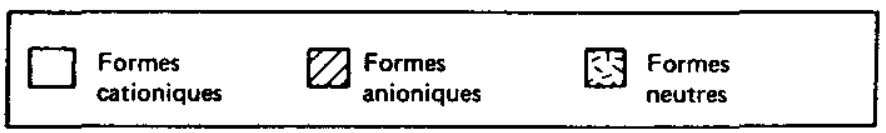

Figure 1.- Bilan ionique moyen du ${ }^{60} \mathrm{Co}$ dissous 3 ou 4 jours après la contamination de 1 'eau.

Figure 2.- Mean ionic balance of dissolved ${ }^{60} \mathrm{Co} 3$ or 4 days following the contcomination of water.

\section{Paramètres relatifs aux carpes}

Le taux de distribution de la nourxiture étant adapté aux besoins des individus, on constate une corrélation étroite entre la température et la croissance pondérale des carpes. Celle-ci est exponentielle dans tous les cas, mais la pente des courbes est d'autant plus forte que la température est élevée (tableau 1). Ce fait se traduit par un taux de grossissement. journalier multiplié par 30 entre $8^{\circ} \mathrm{C}$ et $25^{\circ} \mathrm{C}$, soit respectivement : 2,4 et $73 \mathrm{mg} \cdot \mathrm{j}^{-1}$. 
La relation entre température et croissance pondérale des carpes contribue à expliquer les données évoquées précédemment et qui concernent. l'évolution des formes ioniques et la concentration du ${ }^{6}{ }^{6} \mathrm{Co}$ dans I'eau des bacs d'expérimentation. I'apparition des formes anioniques et neutres du radjocobalt peut être imputée, comme le suggèrent FUKAI et MURRAY (1973) ainsi que KILLEY et al., (1984), à une surcharge en matières crganiques liée à la distribution de nourriture et a l'activité métabolique des poissons. Celle-ci étant plus faible à $8{ }^{\circ} \mathrm{C}$, il est logique que les formes cationiques demeurent plus importantes dans ce cas, que pour les trois temperatures plus élevées. De plus, la présence des poissons en milieu confiné entraine une très forte augmentation d'ions tels que $\mathrm{HCO}_{3}{ }^{-}, \mathrm{Cl}^{-}, \mathrm{SO}_{4}--, \mathrm{PO}_{4}{ }^{--}$et $\mathrm{NH}_{4}{ }^{+}$(FRITSCH et BAUDIN, 1984) qui peuvent $\mathrm{s}^{\prime}$ associer avec les ions $\mathrm{CO}^{++}$pax des réactions elles-mêmes dépendantes de la température, pour donnex des molécules neutres et des complexes anioniques (TRISCHAN et al., 1981).

En ce qui concerne la radioactivité de l'eau, la décroissance constatée est également liée à la température, par le fait que les fèces. sux lesquelles se fixe fortement le radiocobalt, sont d'al:tant plus abondantes que le métabolisme des poissons est intense. D'autre part, le développement sur les parois des bacs de micro-organismes pouvant participer a la fixation du ${ }^{60} \mathrm{Co}$ est d'autant plus rapide et important que la température est élevée.

Malgré la très grande homogenéité de chacun des 4 lots, 1 'examen des paramètres relatifs à la radiocontamination des carpes révèle des écarts importants entre les valeurs individuelles (tableaux 2 et 3 ). En conséquence, afin d'évaluer I'influence du facteur thexmique avec une fiabilité satisfaisante, des analyses de variance ont été effectuées sur les rếsultats obtenus après envixon 40 jours d'expérience ; les conclusions de ces analyses sont fondées sur un risque d'erreur inférieur à 5 (test de SNEDECOR). Comme 1 'indique le tableau 4 , 1'appréciation de $l^{\prime}$ effet de la tempéxature sur $1^{\prime}$ accumulation du ${ }^{60} \mathrm{Co}$ pax les carpes dépend du paramètre considéré. On constate ainsi des différences marquées selon que l'on se réfère à la radioactivité totale des poissons ou à la concentration du radionucléide.

Globalement, la quantité totale de radiocobalt fixée pax les carpes apparalt très fortement liée au facteur thermique mais, selon le niveau des températures, des situations différentes se dégagent pour des mèmes écarts. Entre 8 et $15^{\circ} \mathrm{C}$, par exemple, les valeurs ne diffèrent pas significativement, alors que, pour un écart légèrement inférieur, l'incidence de la temperature est sensible entre 15 et $20^{\circ} \mathrm{C}$ et assez nette entre $20^{\circ} \mathrm{C}$ et $25^{\circ} \mathrm{C}$ (tableau 4). Cette gradation apparait également quand on considère les deux écarts comparables les plus importants que sont $8^{\circ} \mathrm{C}-20^{\circ} \mathrm{C}$ et $15^{\circ} \mathrm{C}-25^{\circ} \mathrm{C}$. Ces observations tendent à indiquer qu'il $n^{\prime} y$ a pas une proportionnalité complète entre l'élévation de température et l'accumulation de ${ }^{60} \mathrm{Co}$ mais une augmentation discontinue de la radioactivité témoignant de l'existence de paliers. $L^{\prime}$ un des paliers les plus importants semble se situer aux environs de $20^{\circ} \mathrm{C}$, température qui constitue la limite inférieure de l'optimum thermique des carpes. La concentration du ${ }^{6} \mathrm{Co}$ dans les poissons est nettement moins influencée par le facteur thermique que la quantité totale accumulée. Ainsi, pour les faibles écarts de températures $\left(5\right.$ et $\left.7^{\circ} \mathrm{C}\right)$, le facteur de concentration est très peu ou pas du tout différent, de mêne entre 8 et $20^{\circ} \mathrm{C}$ qui déterminent pourtant une différence importante. $L$ 'incidence de la température $n^{\prime}$ est vraiment sensible qu'entre 15 et $25^{\circ} \mathrm{C}$ et, a fortiori, entre 8 et $25^{\circ} \mathrm{C}$. Ces données indiquent bien une absence de proportionnalité complète entre l'élévation de température et la concentration du radionucléide et $l^{\prime}$ existence d'un palier se situant vers $20^{\circ} \mathrm{C}$. 
Tableau 2.- Quantité totale de ${ }^{60} \mathrm{Co}$ dans les carpes (Bq). Tableau 3.- Concentration du ${ }^{60} \mathrm{Co}$ dans les carpes (Bq.9 ${ }^{-1}$ ). Table 2.- ${ }^{60} \mathrm{Co} b$ dy burden of carps $(\mathrm{Bq})$. Table 3. ${ }^{60} \mathrm{Co}$ concentration in carps $\left(B q \cdot g^{-1}\right)$.

\begin{tabular}{|c|c|c|c|c|}
\hline \multirow{2}{*}{$\begin{array}{l}\text { Temps } \\
\text { (Jours) }\end{array}$} & \multicolumn{4}{|c|}{ Temperatures } \\
\hline & $8^{\circ} \mathrm{C}$ & $15^{\circ} \mathrm{C}$ & $20^{\circ} \mathrm{C}$ & $25^{\circ} \mathrm{C}$ \\
\hline 1 & $58 \pm 52$ & $165 \pm 79$ & $486 \pm 182$ & $829 \pm 254$ \\
\hline 2 & $71 \pm 60$ & $150 \pm 66$ & $629 \pm 332$ & $769 \pm 124$ \\
\hline 4 & . & - & $446 \pm 199$ & $662 \pm 139$ \\
\hline 5 & $231 \pm 133$ & $313 \pm 189$ & · & - \\
\hline 8 & . & $395 \pm 177$ & $620 \pm 255$ & $1265 \pm 235$ \\
\hline 11 & . & - & $1177 \pm 416$ & $1444 \pm 177$ \\
\hline 12 & $762 \div 570$ & $454 \div 109$ & . & - \\
\hline 15 & - & . & $1450 \pm 544$ & $1256 \pm 222$ \\
\hline 19 & $880 \pm 710$ & $415 \pm 113$ & . & . \\
\hline 22 & . & . & $1468 \pm 334$ & $1567 \pm 288$ \\
\hline 27 & $703 \pm 448$ & $299 \pm 81$ & - & . \\
\hline 29 & . & - & $1773 \pm 455$ & $2745 \pm 561$ \\
\hline 33 & $921 \pm 339$ & $612 \pm 281$ & - & . \\
\hline 36 & - & . & $1813 \pm 558$ & $2752 \pm 555$ \\
\hline 40 & $624 \pm 234$ & $612 \pm 261$ & - & • \\
\hline 43 & - & . & $1969 \pm 597$ & $3121 \pm 1051$ \\
\hline 47 & $704 \pm 293$ & $900 \pm 499$ & - & - \\
\hline 49 & . & . & . & $2913 \pm 721$ \\
\hline .54 & $865 \pm 475$ & $997 \pm 469$ & . & . \\
\hline
\end{tabular}

\begin{tabular}{|c|c|c|c|c|}
\hline Temps & & Temp & Gratures & \\
\hline (Jours) & $\mathrm{g}^{\circ} \mathrm{C}$ & $15^{\circ} \mathrm{C}$ & $20^{\circ} \mathrm{C}$ & $25^{\circ} \mathrm{C}$ \\
\hline 1 & $24 \pm 19$ & $77 \pm \quad 39$ & $220 \pm 74$ & $331 \div 98$ \\
\hline 2 & $31 \pm 27$ & $71 \pm 31$ & $291 \pm 132$ & $325 \pm 53$ \\
\hline 4 & . & - & $186 \pm 81$ & $251 \pm 51$ \\
\hline 5 & $101 \pm 60$ & $141 \pm 84$ & - & - \\
\hline 8 & . & $171 \div 76$ & $253 \pm 102$ & $456 \geq 81$ \\
\hline 11 & . & . & $448 \pm 160$ & $495 \pm 54$ \\
\hline 12 & $296 \div 206$ & $189 \pm 44$ & - & - \\
\hline 15 & - & . & $518 \pm 196$ & $390 \pm 58$ \\
\hline 19 & $336 \pm 262$ & $161 \div 49$ & . & . \\
\hline 22 & . & . & $446 \pm 104$ & $414 \pm 64$ \\
\hline 27 & $272 \pm 148$ & $114 \pm 32$ & . & - \\
\hline 29 & - & - & $468 \pm 129$ & $602 \pm 121$ \\
\hline 33 & $352 \pm 141$ & $221 \div 98$ & . & . \\
\hline 36 & . & - & $433 \pm 135$ & $553 \pm 104$ \\
\hline 40 & $246 \pm 81$ & $230 \pm 104$ & . & . \\
\hline 43 & . & - & $445 \pm 135$ & $607 \pm 137$ \\
\hline 47 & $271 \pm 97$ & $304 \pm 178$ & . & . \\
\hline 49 & . & - & - & $492 \pm 123$ \\
\hline 54 & $332 \pm 173$ & $331 \pm 163$ & . & . \\
\hline
\end{tabular}


Tableau 4.- Influence de la température sur les différents paramètres relatifs à 1 accumulation du ${ }^{60} \mathrm{Co}$ par les carpes

$(0=$ influence nulle; $+=$ influence positive $)$.

Table 4:- The effect of temperature on the various parameters related to the ${ }^{60} \mathrm{Co}$ accumulation by carps

$(0=$ no effect $;+=$ positive effect $)$.

\begin{tabular}{cccc}
\hline $\begin{array}{c}\text { Ecarts } \\
\text { dempératures }\end{array}$ & \multicolumn{3}{c}{ Paramètres relatifs au ${ }^{60} \mathrm{Co}$} \\
\cline { 2 - 4 } & $\begin{array}{c}\text { Quantité } \\
\text { totale }\end{array}$ & Concentration & $\begin{array}{c}\text { Facteur de } \\
\text { concentration }\end{array}$ \\
\hline $8^{\circ} \mathrm{C}-15^{\circ} \mathrm{C}$ & 0 & 0 & 0 \\
$15^{\circ} \mathrm{C}-20^{\circ} \mathrm{C}$ & +++ & + & + \\
$20^{\circ} \mathrm{C}-25^{\circ} \mathrm{C}$ & + & 0 & 0 \\
$8{ }^{\circ} \mathrm{C}-20^{\circ} \mathrm{C}$ & +++ & + & + \\
$15^{\circ} \mathrm{C}-25^{\circ} \mathrm{C}$ & ++++++ & ++ & ++ \\
$8{ }^{\circ} \mathrm{C}-25^{\circ} \mathrm{C}$ & +++++++ & ++ & ++ \\
\hline
\end{tabular}

- Valeurs à 40 jours pour 8 et $15^{\circ} \mathrm{C}$, à 36 jours pour 20 et $25^{\circ} \mathrm{C}$.

L'évolution du facteur de concentration est similaire pour les 4 températures et se caractérise par deux phases successives : la première correspond à une élévation rapide de la valeur du paramètre et la seconde à un accroissement très lent conduisant à un état d'équilibre. Cette évolution peut se traduire par un modèle exponentiel (figuxe 2), fondé sur l'existence, dans les organismes, de plusieurs compartiments caractérisés par les vitesses des flux entrant et sortant de l'élément considéré (GOLDSTEIN et ELWOOD, 1971 ; AOYAMA et INOUE, 1973 ; PENTREATH, 1975 ; GONTIER, 1983). D'après les résultats obtenus dans nos expériences, ce modèle permet de mettre en évidence deux compartiments pour les lots maintenus a $8^{\circ} \mathrm{C}$ et $15^{\circ} \mathrm{C}$, alors que pour les 2 températures les plus élevées le transfert correspond à un modèle tricompartimental.

Les équations représentatives de l'évolution du facteur de concentration permettent, par extrapolation, de calculer la valeur maximale du paramètre ainsi que le temps nécessaire à $I^{4}$ instauration de $1^{\prime}$ état d'équilibre correspondant à cette valeur. Pour les 4 températures, ces deux données sont de 3,6 et 310 jours pour $8^{\circ} \mathrm{C}, 4,4$ et 220 jours pour $15^{\circ} \mathrm{C}, 5,1$ et 125 jours pour $20^{\circ} \mathrm{C}$ et enfin, 6 et 110 jours pour $25^{\circ} \mathrm{C}$. On constate que l'élévation de la température se trađuit par une augmentation de la valeur maximale du facteur de concentration et une diminution de la durée de la phase conduisant à l'état d'équilibre. Cette relation entre le facteur thermique et la concentration du ${ }^{\circ}{ }^{\circ} \mathrm{Co}$ peut être liée au fait que l'accroissement de température entrainant une baisse de l'oxygène dissous (loi de Henry), les poissons augmentent leur circulation interne d'eau afin d'extraire une même quantité d'o2 nécessaire à leur respiration. Ce mécanisme compensatoire peut, d'après RAMADE (1978), favoriser la fixation des matières polluantes présentes dans le milieu. DEMAEL et GARCIN (1978) estiment que l'augmentation de la temperature de 1'eau favorise, chez les poissons, les échanges avec le milieu extérieur et stimule la plupart des processus métaboliques, ce qui pourrait induire des phenomènes d'accumulation intracellulaire de certaines substances (ROMANENKO, 1984). 

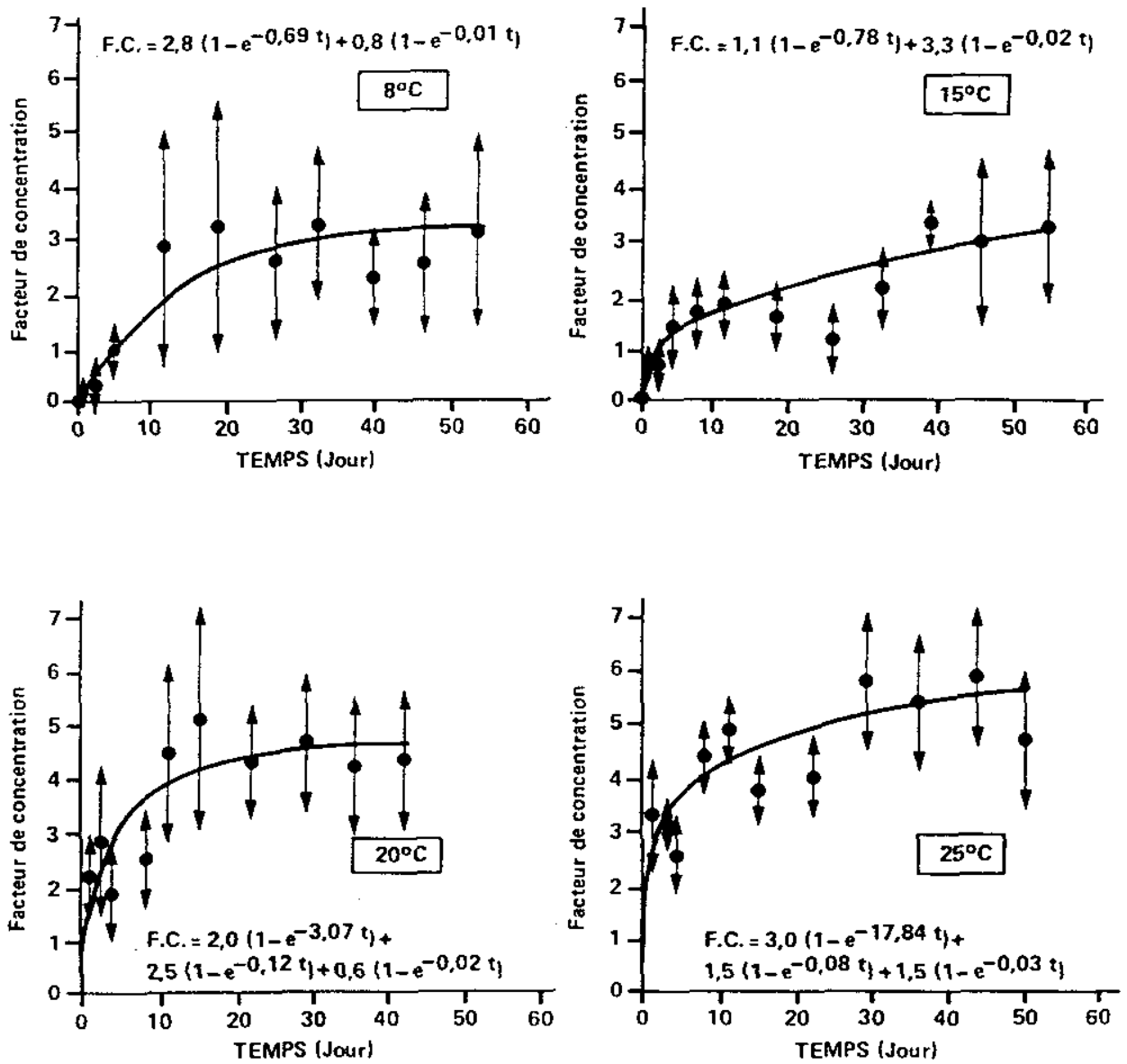

Figure 2.- Evolution du facteur de concentration du ${ }^{6}{ }^{0} \mathrm{Co}$ par les carpes en fonction de la temperature (valeurs moyennes affectées de l'intervalle de confiance $t . s / \sqrt{n}$ où " $t$ " est la variable de student pour 95 \& de probabilité, " $s$ " la déviation standard estimée et " $\mathrm{n}$ " le nombre d'individus).

Figure 2.- Development of ${ }^{60} \mathrm{Co}$ concentration factor by carp as a flonction of temperature (mean values given with their confidence interval $t \times s / \sqrt{n}$, where " $t$ " is the student variable for $95 \%$ probability, " $s$ " the estimated standard deviation and " $n$ " the number of individuals).

Dans une étude fondée sux une analyse bibliographique et consacrée à l'influence de la temperature sur la radiocontamination des organismes aquatiques, AMIARD et KHALANSKI (1981) ont utilise le coefficient thermique $Q_{10}$ comme relation entre l'échauffement de $I^{\prime}$ eau et la radiocontamination. Ce coefficient, fondé sux 1'hypothèse selon laquelle la vitesse des processus physiologiques est une fonction exponentielle de la température, se définit ainsi :

$$
Q_{10}=\left(\frac{C_{1}}{C_{2}}\right) \frac{10}{T_{1}-T_{2}}
$$


où $C_{1}$ et $C_{2}$ sont les valeurs du facteur de concentration mesurées aux températures $T_{1}$ et $T_{2}$. Polir $l^{\prime}$ ensemble des radionucléides étudiés, AMIARD et KHALANSKI (1981) estiment un coefficient moyen de 2,4 pour les poissons, alors qu'il est compris en général entre 2 et 3 pour de nombreux processus physiologiques et qu'il varie avec la temperature. Il faut signaler que cette valeur est en accord avec les données de LEVESQUE (1980) qui indlque un $Q_{10}$ variant entre 2 et 3 pour beaucoup de réactions métaboliques chez les organismes vivant dans un milieu soumis à des rejets d'eaux chaudes. Or, appliqué au facteur de concentration calculé pour les 4 lots de carpes, le $2_{10}$ est toujours nettement inférieur à 2, pour tous les écarts de température considérés, que I'évaluation soit faite d'après les valeurs obtenues après 40 jours d'expérience $\left(Q_{10} \propto 1,7\right)$ ou d'après les valeurs maximales théoriques correspondant à $l^{\prime}$ instauration de l'état d'équilibre $\left(Q_{10} \simeq 1,3\right)$. On constate, tout d'abord, que le coefficient thermique est donc largement inférieur à celui cité pax AMIARD et KHALANSKI (1981), calculé, il est vrai, d'après des données bibliographiques qui ont probablement été évaluées dans des conditions très variées. Sur un plan plus général, nos résultats tendent à montrer que, chez les poissons, la vitesse des mécanismes impliqués dans 1 'accumulation du ${ }^{60}$ Co $n^{\prime}$ 'est pas proportionnelle à l'élévation de tempéxature comme c'est généralement le cas pour les processus physiologiques. En conséquence, la corrélation entre l'élévation de la tempéxatuxe et l'augmentation de la quantité totale de ${ }^{60} \mathrm{Co}$ fixé apparaît donc plus étroitement liée à un accroissement du pool de cobalt, dû à la croissance pondérale des individus, qu'à une intensification du métabolisme. Par contre, ce phénomène est sans doute responsable d'une légère augmentation du taux de renouvellement du cobalt, auquel peuvent etre imputées les faibles différences entre les valeurs du facteur de concentration, si on admet que pour une teneur normale de $l^{\prime}$ eau en cobalt, $l^{\prime}$ accumulation du ${ }^{60} \mathrm{Co} \mathrm{s}^{\prime}$ effectue par remplacement de l'isotope stable.

\section{CONCLUSION}

Diverses espèces de poissons dulçaquicoles et notamment Cyprinus carpio, ont fait 1 'objet de travaux approfondis qui ont mis en evidence l'incidence déterminante đu facteur thermique sur les activités métaboliques (LEVESQUE, 1980). Comme le démontrent nos résultats, la température $n^{\prime} a$ pas un rôle aussi prépondérant sur 1 'accumulation du ${ }^{6}{ }^{\circ} \mathrm{Co} \mathrm{chez}$ Cyprinus carpio, mais elle représente, toutefois, un facteur qui influe sensiblement sur la contamination des poissons pax ce radionucléide.

Chez des carpes présentant une croissance pondërale suffisamment importante, les augmentations concomittantes de la quantité totale de ${ }^{60}$ Co fixé et de la température ne peuvent rendre compte de l'influence réelle du facteur thermique sur les processus physiologiques dont résultent l'accumulation du radionucléide. En effet, l'accroissement du pool total de cobalt, consécutif au gain de masse des individus, suffit à expliquer l'augmentation de la quantité de radiocobalt fixé par échange isotopique. A l'inverse, I'analyse des valeurs du facteur de concentration permet de conclure que les variations de temperrature influent sur la fixatior: du ${ }^{60} \mathrm{co}$ par la carpe, mais qu'il n'y a pas proportionnalité entre les fluctuations thermiques et la radiocontamination. Ainsi, on constate une influence nulle ou très faible pour les écarts de températures inférieurs 
à $10^{\circ} \mathrm{C}$ ou quand la température maximale ne dépasse pas $20^{\circ} \mathrm{C}$. L'incidence du facteur thermique devient sensible pour des écarts supérieurs à $10^{\circ} \mathrm{C}$ et notamment quand les températures atteignent les valeurs correspondant à l'optimum thermique des carpes. Sux le plan de la protection sanitajre, on peut déduire de ces études que le réchauffement des eaux ne constitue pas un facteur majeur d'intensification de la radiocontamination des poissons pax le ${ }^{60} \mathrm{Co}$, même si celui-ci provoque une augmentation du facteur de concentration et de la vitesse d'accumulation du radiocobalt. Le triplement de la température, correspondant au passage d'un seuil au-dessous duquel les carpes ne se nourrisent plus $\left(8^{\circ} \mathrm{C}\right)$, à un niveau représentant $1^{\prime}$ optimum thermique des carpes $\left(25^{\circ} \mathrm{C}\right)$, se traduit, à l'équilibre, par un facteur de concentration évoluant seulement de 3,6 à 5,1 . Un faible pool de cobalt et un coefficient élevé de renouvellement (BAUDIN et FRITSCH, 1987) peuvent justifier ces faibles valeurs maximales et $l^{\prime}$ 'instauration rapide de l'état d'équilibre, contrairement à ce que l'on constate, avec la même espèce, pour d'autres radionucléides, tels que $1 e^{65} \mathrm{zn}$, le ${ }^{54} \mathrm{Mn}$ ou le ${ }^{22} \mathrm{Na}$ (BAUDIN, 1983 ; FRITSCH et BAUDIN, 1984).

\section{REMERCIEMENTS}

Ce travail s'inscrit dans un programe de recherche faisant l'objet d'un contrat entre le Commissariat à l'Energie Atomique et la Commission des Conmunautês Européennes intitulé "Etude comparative de la rađioécologie des eaux du Rhône et de la Meuse". Nous remercions vivement cette commission pour toutes les possibilités de coopération offertes par une étude de cette ampleur.

\section{RÉFÉRENCES BIBLIOGRAPHIQUES}

AMIARD J.C., KHALANSKI M. (1981). Conséquence de l'élévation de la température de l'eau sur la radiocontamination des organismes aquatiques. Ann. Biol, , 20(2): 115-130.

AOYAMA I., INOUE Y. (1973). Estimation and evaluation of radioactive contanination through a food web in an aquatic ecosystem. An application of the compartment model to transfer of radioactive contamination through a food web in an aquatic ecosystem. An application of the compartment model to transfer of radio- active substance through a food chain. J. Radiat. Res., 14(4): 375-381.

BAFDIN B., BERTIN M., GUIEZE J.L. (1982). Radioactive materials release from nuclear plant in France : In : Radionuclide metabolism and tcxicity, 302-304, paris, Masson.

BAUDIN J.P. (1983). Etude experimentaie de la bioaccumulation et de l'excretion du ${ }^{6} \mathrm{zn}$ par un poisson dulçaquicole, Cyprinus carpio L. Acta Oecologica. Oecol. Applic., 4(2): 139-149. 
BAUDIN J.P., FRITSCH A.F. (1987) . Retention of ingested ${ }^{60} \mathrm{Co}$ by a freswater fish. Water, Air, and Soil Pollut., 36: 207-217.

CANTILLON G., KIRCHMANN R.

VAN DER BORGHT O. (1969). F1xation de radiomanganèse et de radiocobalt par les sédiments, la faune et la flore dans la Meuse en aval de la centrale franco-belge des Ardennes. In : Actes du 1er Symp. Internat. de Radioeculogie, Cadarache, 8-12 September 1969, Cadarache, CEA, 127-163.

COLE J.C., CARSON B.L. (1981). CObalt in the food chain. In : Trace metals in the environment, edited by Smith I.C. and Carson B.L., vol. 6 - Cobalt, 777 - 924. Ann Arbor, Ann Arbor Science.

COUGHTREY P.J., THORNE M.C. (1983). Radionuclide distribution and transport in terrestrial and aquatic ecosystems. Rotterdam, Balkema vol, 2, 500 p..

D'AUBENTON F. SPILLMANN C.J. (1976). La carpe sauvage, Cyprinus carpio Linné. La pisciculture francaise, 45: 59-62.

DEMAEL A., GARCIN D. (1978). Effets d'un choc, thermique de $10^{\circ} \mathrm{C}\left(15-25^{\circ} \mathrm{C}\right)$ sur certains parametres metaboliques de $1 \mathrm{a}$ tanche adaptée a des eaux diversement oxygéneses. Cahiers du Laboratoire de Montereau, 7: 15-27.

DEsSAIX J. (1980). Les gammares du Rhơne en amont de Iyon. Dynamique des populations et estimation de production. These Doct. Spécialite, Univ. Lyon I, $129 \mathrm{p.}$

FOULOUIER L., PALLY M. (1982), Données sux la teneur en tritium l1e de poissons de grands fleuves français. Annales de $Z$ 'Association Belge de Radioprotection, 7(3-4): 259-281.

FOULQUIER L., PALLY M. (1984). Données radioécologiques sur les sédiments du bas Rhône. Revue Française des Sciences de Z'Eou, 3(3): 259-277.

FOULQUIER L., DESCAMPS B., BAUDIN-JAULENT Y. (1985). Mesure et interprétation des teneurs en radionucléldes des polssons du haut-Rhône français. Verh. Internat. Verein. Limnol., 22: 2470-2475.

FRITSCH A.F., BAUDIN J.P. (1984), Etude experimentale de I'absorption et de la désorption par la carpe lCyprinus carpio $\left.\mathrm{L}_{\mathrm{i}}\right]$ d'un melange de ${ }^{6} \mathrm{Co},{ }^{3} \mathrm{Cr},{ }^{13}{ }^{7} \mathrm{Cs}$, ${ }^{54} \mathrm{Mn}$ et ${ }^{22} \mathrm{Na}$. Rapport CEA - R - 5251, Saclay, CBA, 59 p..
FUKAI R., MURRAY C.N. (1973). Environmental behaviour of radiocobalt and radiosilver released from nuclear power stations into aquatic systems. In : Environmental behaviour of radionuclides released in the nuclear industrie.

Proceed of a Symp., Aix-en-Provence (Erance), May 14-18, 1973, 217-242, Vienne, AIEA.

GOLDSTEIN R.A., ELWOOD J.W. (1971). A two compartment, three parameters model for the comparison absorption and retention of ingested elements by animals. Ecology, 52: 935-939.

GONTIER G. (1983), Le transfert des radionucléides aux organismes aquatiques. La démarche expérimentale et son application a l'étude de la bioaccumulation du cobalt 60 , du manganèse 54 et du chrome 51 pax Sparus amatus, Nephrops norvegicus et Hommarus gammarus. Thèse Doct. Jème cycle, univ. Aix-Marseille II, 66 p..

HUET M. (1970). Traité de pisciculture. Bruxelles, Ch. de Wyngaert, $718 \mathrm{p}$.

KAYE s.U. (1973). Assessing potential radioecological impacts of aquatic bjota in response of the National Environmental Pollcy Act of 1969. In : Environmental behaviour of radionuclides released in the nuclear industry. Proceed of a Symp. Aix-en-Provence (Fronce), May 1418, 1973, 649-663, Vienne, IAEA.

KILLEY R.W.D., MC HUGH J.D., CHAMP D.R., COOPER E.L., YOUNG J.L. (1984). SUb surface cobalt 60 migration from a low-level waste disposal site. Environ. Sci. Technol., 18(3): 148-157.

LACOMBE C., CREACH Y. (1974). InEluence d'une elevation de temperature sur quelques parametres physiologiques en relation avec le metabolisme azote et hydrothermal de la carpe. Cahiers du Laboratoire d'Hydrobiologie de Montereau, 1: $71-84$

LEVESOUE L, (1980), Les rejets d'eaux chaudes des centrales thermiques. I : Etudes sur sites des effets sur la vie aquatique. Paxis, Association Française pour 1'Etude des Eaux, 177 p..

MYCHOLET-COTE C., XIRCHMANN R., CANTILLON R. , COLARD J., KOCH G. (1973). Etude de la radiocontamination des poissons de la Meuse. In : Environmental behaviour of radionuclides released in the nuclear industrie. Proceed of a Symp., Aix-enProvence (France), May 14-18, 1973, 413-427, Vienne, AIEA. 
MURAT J.C., PARENT J.P. (1984). Effets de chocs thermiques sur le métabolisme glucidique de la carpe. Cahiers du Laboratoire d'Hydrologie de Montereau, 1: 85-94.

MUUS B.J., DALHSTROM P. (1968). Guide des poissons $d^{\prime}$ eau dunce et pêche. Neufchătel, Delachaux et Niestle S.A., 242 P..

PENTREATH R.J. (1975). Radiobiological studies with marine fish. In : Design of radiotracer experiments in mamine biologicat systems, 137-170. Technical. Reports series, $n^{\circ} 167$, Vienne, AIEA.

PHILIIPS G.R., RUSSO R.C. (1978). Metal bioaccumulation in fishes and aquatic invertebrates : a literature review. U.S. Environmental Protection Agency, Report $N^{\circ}$ EPA 600/3,78-103, 115 p..
PICAT Ph., BOURDEAU F., THIRION J.P., SIGALA M., QUINAULT J.M., ARNAUD M., CARTIER Y. (1985), Study of the physiccchemical form of cobalt in the Loire River. In : Speciation of fission and activation products in the environment, edited by Bulman R.A. and Cooper J.R., 269-285, London and New-York, Elsevier Applied Science Publishers.

RAMADE F. (1978). Elements d'ecologie appliquée. Paris, Ediscience, Mc GrawHill, 576 p..

ROMANENKO V.D. (1984). Metabolic aspect of warmwater culture of the carp. Cyprinus carpio L., Aquaculture, 38: 307-314.

TRISCHAN G.M., WILKINSON R.R., CARSON B.L. (1981). Chemistry of cobalt. In : Trace metals in the environment, edited by Smith I.C. and Carson B.L., vol. 6 , cobalt, 229-370, Ann Arbour, Ann Arbour science. 